\title{
Magnetic field modelling from scalar-only data: Resolving the Backus effect with the equatorial electrojet
}

\author{
R. Holme ${ }^{1}$, M. A. James ${ }^{1 *}$, and H. Lühr² \\ ${ }^{1}$ Department of Earth and Ocean Sciences, University of Liverpool, 4, Brownlow St., Liverpool, L69 3GP, U.K. \\ ${ }^{2}$ GeoForschungsZentrum Potsdam, Telegrafenberg, D-14473 Potsdam, Germany
}

(Received October 15, 2004; Revised August 8, 2005; Accepted August 12, 2005)

\begin{abstract}
It is well known that models of the global geomagnetic field constructed from only measurements of the field intensity suffer from large errors arising from the Backus or perpendicular error effect. Knowledge of the location of the magnetic dip equator is in principal sufficient to eliminate this error. We investigate constraining the location of the dip equator using observations of the equatorial electrojet in intensity measurements made from the CHAMP satellite. While the models generated are inferior compared with models obtained from oriented three-component vector data, they may be of sufficient quality to allow construction of future global geomagnetic reference models in the absence of vector data.
\end{abstract}

Key words: Geomagnetic field modelling, equatorial electrojet, Backus effect.

\section{Introduction}

Modelling of the main geomagnetic field relies on the availability of high-quality, well-distributed magnetic measurements. The easiest way to achieve uniform coverage is through a low Earth-orbiting satellite flying in a polar orbit. With this motivation, the POGO series of satellites were flown in the mid to late 1960s and early 1970s (Cain and Langel, 1971; Langel, 1974), measuring the total field strength but not its direction. However, field models derived from these data were compromised by large errors: it became clear that measurements of field intensity alone were insufficient to produce an accurate field model (e.g., Benkova et al., 1971). Backus (1970) proved that for a primarily dipole magnetic field, inversion from intensity data alone is formally non-unique, as two nontrivially different potential fields yield the same intensity everywhere on a spherical surface. Stern and Bredekamp (1975) demonstrated that this formal non-uniqueness was closely related to the discrepancies in the POGO field models. Lowes (1975) described the link between the two particularly clearly, in terms of the perpendicular error effect. The non-uniqueness discovered by Backus (1970) arises from a potential field that is everywhere perpendicular to a dipole field. However, to first order, the effect on magnetic intensity of a small perturbation to the main field is the component of the perturbation field in the direction of the main field. Therefore, intensity measurements are insensitive to fields of the form of the Backus formal non-uniqueness. A clear demonstration of the Backus effect was provided us-

*Now at: School of Earth \& Environment, University of Leeds, Leeds, LS2 9JT, U.K.

Copyright (c) The Society of Geomagnetism and Earth, Planetary and Space Sciences (SGEPSS); The Seismological Society of Japan; The Volcanological Society of Japan; The Geodetic Society of Japan; The Japanese Society for Planetary Sciences; TERRAPUB ing MAGSAT data by Stern et al. (1980).

Considerable effort has been devoted to finding a method by which satellite intensity data could be used to produce high-quality field models. It was found that including vector component data from magnetic observatories, especially close to the equator, would significantly reduce the effect of the non-uniqueness (Hurwitz and Knapp, 1974; Barraclough and Nevitt, 1976; Gubbins and Bloxham, 1985; Lowes and Martin, 1987; Ultré-Guérard and Achache, 1997). This observation was explained by Khokhlov et al. (1997), who demonstrated that, in principal, the Backus effect could be eliminated with knowledge of the position of the geomagnetic dip equator, the points on the Earth's surface at which the vertical component of the field is zero. Ultré-Guérard et al. (1998) developed this result into a field modelling strategy. They suggested that beginning from an epoch when the field is constrained by vector data, a model of secular variation could be used to estimate the change in position of the dip equator through time. This position could then be combined with satellite intensity measurements to calculate a field model. Unfortunately, secular variation models depend crucially on magnetic observatory measurements; as observatories are unevenly distributed, with particularly few near the equator, the secular variation model and hence the dip equator position derived from it is compromised.

However, the position of the dip equator can be obtained in another way. Main field modelling has typically made use of only nightside satellite observations, as dayside observations are more strongly contaminated with fields from ionospheric currents. One particularly strong dayside signal comes from the equatorial electrojet, a current system flowing in the ionosphere along the dip equator, seen particularly strongly near local noon (Onwumechili, 1997). This current system generates a drop in the field intensity above the ionosphere of up to a few tens of nT, with a sharp peak 
in the anomaly very close to the dip equator. Therefore, this signal might be used to determine the position of the dip equator at ionospheric height. This leads us to investigate the following field-modelling strategy. We consider a low Earth-orbiting satellite in noon-midnight orbit, and combine nightside measurements of field intensity with dayside observations of the peak in the signal from the equatorial electrojet. These positions are incorporated into the field modelling as zero measurements of the vertical field, located at $6486 \mathrm{~km}$ geocentric radius, assuming an approximate height of the electrojet of $108 \mathrm{~km}$ above the geographic equator (following Lühr et al., 2004). In principle, this combination of measurements should allow the determination of a global field model using satellite intensity measurements alone. Here we test whether the measurements are sufficient in practice.

\section{The Position of the Electrojet}

Our proposed strategy relies crucially on how accurately the electrojet defines the dip equator. Jadhav et al. (2002) studied the equatorial electrojet using data from the Ørsted satellite (Neubert et al., 2001). They argued that the electrojet ran close to the equator, but showed departures in latitude from that position of up to a degree. This result was contested by Lühr et al. (2004), who modelled the ionospheric current systems generating the electrojet to fit nearnoon-time observations from CHAMP data (Reigber et al., 2002). They observed no systematic departure of the electrojet peak position from the dip equator. How close the peak position is to the geomagnetic equator is crucial. For ionospheric science, a deviation of only 0.1 degree might be considered to be zero within observational errors, but such a deviation is large for modelling the main field. That field is dominated by its axial dipole component, giving an approximate value for the vertical component at Earth's surface of

$$
Z=-2 g_{1}^{0} \cos \theta
$$

where $g_{1}^{0}$ is the axial dipole Gauss coefficient (approximately $-30,000 \mathrm{nT}$ ) and $\theta$ is colatitude. Near the equator $\left(\theta=90^{\circ}\right)$, the rate of change of $Z$ with colatitude is approximately $1000 \mathrm{nT}$ per degree. An offset in the magnetic equator of $0.1^{\circ}$ would lead to an error in the assumed vertical component of order $100 \mathrm{nT}$; a systematic difference as reported by Jadhav et al. (2002) would produce a large systematic error in any field model, probably worse than the Backus effect itself.

We therefore began by conducting an extensive study of whether the electrojet peak position coincides with the dip equator. Full details are given in James (2004), obtainable on request from the first author; here we summarise only the conclusions. James (2004) used CHAMP satellite data between 1/9/2001 and 5/11/2003 from the scalar Overhauser magnetometer, considering orbits with an equatorial crossing between 11:00 and 13:00 local time, at magnetically quiet times, defined by choosing $\mathrm{Kp}<2-$, and also excluding times of rapid Dst variation. From each pass, she subtracted a high quality model of the large scale field, the CO2003 model, described by Holme and Olsen (2004). A sample orbit after performing this subtraction is shown in Fig. 1. The signal of the equatorial electrojet is clearly

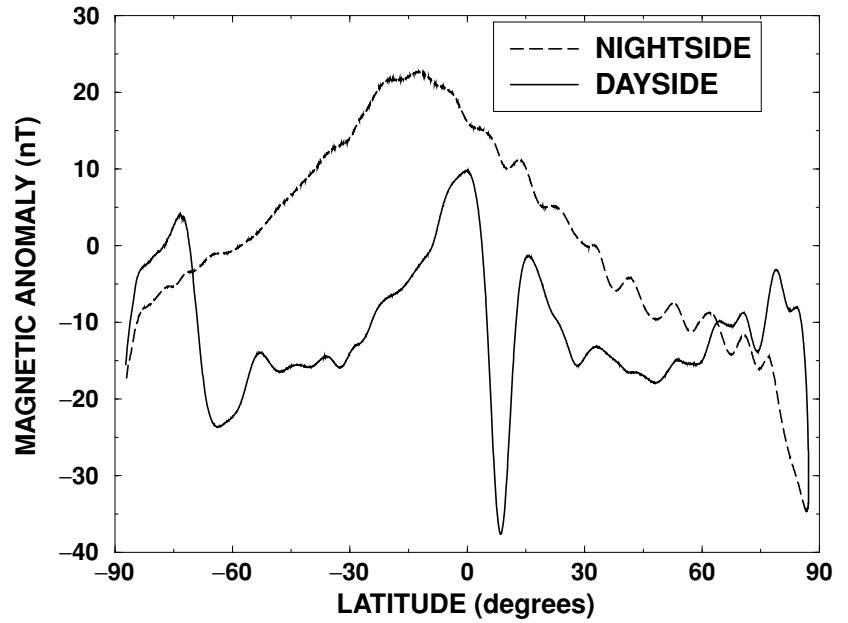

Fig. 1. A typical orbit with both day and nightside data after subtracting the CO2003 field model.

visible in the day side data, with peak at about 10 degrees latitude: this peak clearly distinguishes the day and nightside passes. However, the night-side data show clearly that the CO2003 field model does not explain all of the longwavelength field. Despite parametrisation for external field (including a Dst dependence), a large long-wavelength signal remains, associated primarily with unmodelled external field. If this signal is not accounted for, it can result in an apparent displacement of the electrojet peak position. James (2004) investigated two approaches to this problem. Firstly, she subtracted a linear trend from the data, chosen to make the two side lobes on either side of the electrojet peak of equal strength. Secondly, she applied the filter of Maus et al. (2002), fitting an internal and external dipole field to the data between $\pm 50^{\circ}$ latitude excluding the latitudes of the electrojet signal. Both of these methods improved the fit of the electrojet peak position to the dip equator. After relocation, some evidence was found of systematic displacement of the noontime electrojet peak from the position of the dip equator at $108 \mathrm{~km}$ altitude, but considerably less than reported by Jadhav et al. (2002). It seems likely that their offset arose from the use of the main field model IGRF 8th generation (revised 1999) (Mandea and Macmillan, 2000) for epoch 2000, which does not place the dip equator sufficiently accurately (Lühr et al., 2004). To quantify the fit to the dip equator, the vertical field predicted by $\mathrm{CO} 2003$ was calculated at geocentric radius $6486 \mathrm{~km}$ and the position of the electrojet peak. Were the peak to lie directly on the dip equator, this value should be $0 \mathrm{nT}$. However, in practice, a mean value of almost $150 \mathrm{nT}$ resulted, with a variance of order $200 \mathrm{nT}$. Such large deviations are not unexpected. Our theory (that the anomaly peak at satellite altitude matches exactly the path of the electrojet at 108 $\mathrm{km}$ altitude) is over-simplified, and the presence of other external field signatures can corrupt the determination of the peak position, particularly as, on the scale of fractions of a degree, the peak is not sharp. A fundamental limitation in precision also arises because CHAMP orbits the Earth once every 90 minutes, a rate of four degrees of latitude per minute. The Overhauser magnetometer returns data at ap- 
proximately $1 \mathrm{~Hz}$; therefore, localisation of the dip equator to much better than 0.1 degree (and hence $100 \mathrm{nT}$ ) is unlikely.

\section{Main Field Modelling}

Is this accuracy sufficient to allow main field modelling from satellite intensity data alone? It would be possible to use simulations to address this question. However, Voorhies et al. (1999) have pointed out that such simulations tend to grossly underestimate Backus effect. Instead, we model from CHAMP data, taking six quiet days from September 6-11, 2001. The local time of the equator crossings for these six days is between midnight and 00:30 on the nightside pass, and between midday and 12:30 on the dayside. The Kp index is generally low: we exclude times when it exceeds $1+$ for modelling with the nightside data (this excludes six 3 hour periods between midday 6/9/2001 and midday 12/9/2001). We subsample the nightside data randomly to obtain an approximately equal area distribution, for a total of 3837 data locations.

We attempt to fit the data with a model of the internal magnetic field only. The magnetic field originating from within the Earth measured at the Earth's surface and above can be represented as the gradient of a scalar potential $\mathbf{B}=$ $-\nabla \Phi$ satisfying Laplace's equation $\nabla^{2} \Phi=0$. In spherical geometry, the solution to this equation can be expressed most conveniently in terms of spherical harmonics

$\Phi=a \sum_{l=1}^{\infty}\left(\frac{a}{r}\right)^{l+1} \sum_{m=0}^{l} P_{l}^{m}(\cos \theta)\left(g_{l}^{m} \cos m \phi+h_{l}^{m} \sin m \phi\right)$

Here, $(r, \theta, \phi)$ are spherical coordinates given by distance from the centre of the Earth, colatitude and longitude, $a$ is the radius of the Earth (taken as $6371.2 \mathrm{~km}$ ), and $P_{l}^{m}$ are Schmidt semi-normalised associated Legendre functions in $\cos \theta$, of degree $l$ and order $m .\left\{g_{l}^{m}, h_{l}^{m}\right\}$ are the set of Gauss coefficients which parameterise the field, which we solve for by simple least-squares fit to the data, truncating the potential field equation (Eq. (2)) at degree $l=13$.

To obtain a benchmark for modelling accuracy, we begin by modelling with vector data. CHAMP carries a triaxial fluxgate magnetometer, which measures three orthogonal components of the vector field, and two star cameras which provide the orientation of the satellite. To orient the vector data requires in addition the rotational transformation between the magnetometer and star cameras, described by a set of rotational Euler angles. These angles change slightly during the mission, and so must be solved for in orbit. We fit CHAMP vector data with an internal field model to harmonic degree 13 , an external field to degree 2 , and Dst dependence in degree 1 internal and external field (for more details of this parameterisation, see Olsen et al., 2000), simultaneously solving for the angular transformation, similar to the method described by Olsen et al. (2003). To limit the effects of high latitude field aligned current systems, polewards of $\pm 50^{\circ}$ geomagnetic latitude we use only the field intensity measurements; this results in a total of 2956 vector data triples and 881 scalar intensity measurements. Then, using these calibrated vector data, we calculate a final model using only internal field harmonics to degree 13 ;

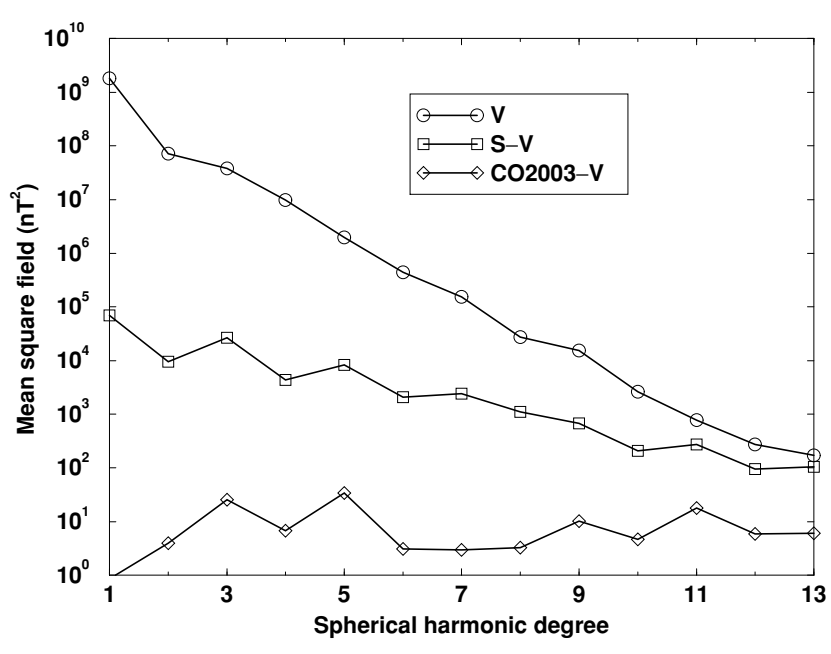

Fig. 2. Power spectra of model V, the difference between model V and model $\mathrm{S}$ (contaminated by Backus effect) and the difference between model $\mathrm{V}$ and the prediction from CO2003, all plotted at Earth radius $(r=6371.2 \mathrm{~km})$

this model, denoted model $\mathrm{V}$, provides the benchmark for our modelling.

Next, we calculate a model from intensity measurements only, solving for internal field only to harmonic degree 13 . As the intensity is a non-linear function of the Gauss coefficients, we must iterate several times to obtain a converged model, denoted model S. As expected, model S shows strong evidence of Backus effect. The error can be quantified by examining its "power spectrum". Various authors (e.g., Mauersberger, 1956; Lowes, 1974) noticed independently that the mean square value of the field, averaged over a spherical surface, has a simple form

$$
\frac{1}{A} \int \mathbf{B}^{2} d A=\sum_{l=1}^{\infty}(l+1)\left(\frac{a}{r}\right)^{2 l+4} \sum_{m=0}^{l}\left(\left(g_{l}^{m}\right)^{2}+\left(h_{l}^{m}\right)^{2}\right) .
$$

where $A$ is the area of the sphere at radius $r$. It is then instructive for a given radius $r$ to plot the individual contributions to this integral from components of different degree $l$ (effectively wavenumber) against that degree. In Fig. 2, we plot the power spectrum at the Earth's surface $r=a$ of model V, and the spectrum of the difference between the two models, giving the total square error in the field model due to the Backus effect at each harmonic degree. We compare this spectrum with that of the difference between model V and the CO2003 model for the same epoch, which quantifies the effects of errors which may be associated with, for example, non-uniform data distribution. The much larger error with the intensity data alone, a consequence of Backus effect, is clear.

\section{Locating Electrojet Peaks}

We now examine whether information from the location of electrojet peaks can reduce the errors seen in model $\mathrm{S}$. We choose to apply the simplest possible method for including this information. Rather than modelling the current system itself, we simply seek the locations of the electrojet peaks in the day side data over the same time interval from which the nightside data were taken. We attempt to 
mimic a true field modelling process as closely as possible. Therefore, we cannot use the CO2003 model to correct for the main field, as were we to have an accurate model of the field, it would obviate the need to model it! Instead, we model the nightside intensity data with a standard least-squares fit, fitting an internal potential field truncated at harmonic degree 14. This model is contaminated by Backus effect, but as the nightside and dayside passes are at effectively the same altitude, this is not important for the correction of the field, as the model provides a good fit to the intensity data at satellite altitude. We combine this model with spherical harmonic degrees 15 and above from the CO2003 model, assuming this to arise from a constant lithospheric field. Correcting for the lithospheric field is most significant in the region of the Bangui crustal magnetic anomaly, which itself could be mistaken for an electrojet peak. We subtract the combined model from the dayside intensity data, and detrend the residual signal by solving track by track for an external and internal (to allow for induced fields) spherical harmonic expansion first to degree 1, and then to degree 2 . In each case, the electrojet peak is then located in two ways: either by picking the minimum point on the trace, or by fitting a quadratic to approximately 20 points nearest the minimum, and adopting the latitude of the minimum of the quadratic. 90 traces yielded peaks in this period. The peak positions obtained with the four different selection methods vary slightly, but the variance between the methods is much smaller than their variance from the true dip equator position. The selected peak locations from degree 1 filtering and fitting with a local quadratic are plotted in Fig. 3. This figure also plots the electrojet peak position profile obtained by Lühr et al. (2004) for MJD700 (modified Julian day defined after 31/12/1999), and the $\mathrm{CO} 2003$ prediction of the dip equator location at a height of $6486 \mathrm{~km}$ at both MJD617 (the epoch of our data set) and MJD700 - the curves for the two epochs are indistinguishable on this plot. The figure shows that the picked electrojet peak positions at satellite altitude follow the location of the true dip equator at the assumed height of the

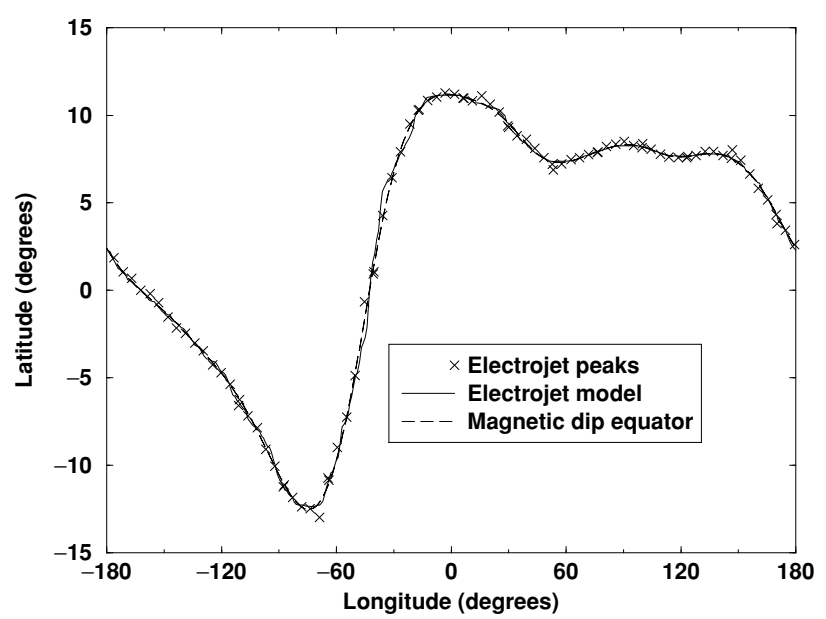

Fig. 3. Electrojet peak positions with degree 1 detrending and fit of quadratic to profile, electrojet model of Lühr et al. (2004), and CO2003 prediction of magnetic dip equator (for both MJD617 and MJD700) at $6486 \mathrm{~km}$. electrojet current system (108 km).

\section{Field Modelling Constrained with the Equato- rial Electrojet}

We calculate four field models, fitting the intensity data and each set of electrojet peak positions. The new models are designated $\mathrm{EE}$ (for equatorial electrojet), 1 or 2 for harmonic degree of filtering, and $\mathrm{P}$ or $\mathrm{Q}$ for picking peaks or fitting a quadratic. For each peak position, we assume that the latitude of the anomaly peak at satellite altitude is the same as the latitude of the electrojet at its nominal height of $108 \mathrm{~km}$ above the equator (approximated as a geocentric radius of $6486 \mathrm{~km}$ ), and define a datum of $0 \mathrm{nT}$ vertical field at this radius. We define a nominal uncertainty of 5 $\mathrm{nT}$ for the scalar data, and $100 \mathrm{nT}$ for $Z=0$ data at the electrojet peak positions. In Fig. 4 we plot the power spectra of the difference of each of these models from model $\mathrm{V}$. In each case, the difference is much less than that from model S (scalar data only), but is still much larger than the difference from $\mathrm{CO} 2003$. Thus, the addition of dip equator information clearly improves the quality of the field model, but by no means sufficiently to replace the need for vector data. In Table 1, we present diagnostics of the models, calculating the RMS difference of each model from model $\mathrm{V}$ averaged over the Earth's surface, the RMS misfit of the model to the scalar data, and the RMS misfit of the model to the $Z=0$ data at electrojet peak positions, followed in brackets by the RMS prediction of model $\mathrm{V}$ at that set of peaks. The improvement over the scalar-data only model $S$ from using information from the electrojet peak positions is clear: the RMS misfit to model V is reduced to less than $20 \%$ of its value from model S. All of the electrojet peak position models give similar results. It is interesting that the degree two field filter does not greatly improve the location of the electrojet peak. This probably arises because our data were recorded in September. The principal signature of degree 2 external field is in the annual variation, which is maximum near the solstices, and close to zero for the epoch of the data we have used. Had we used data from

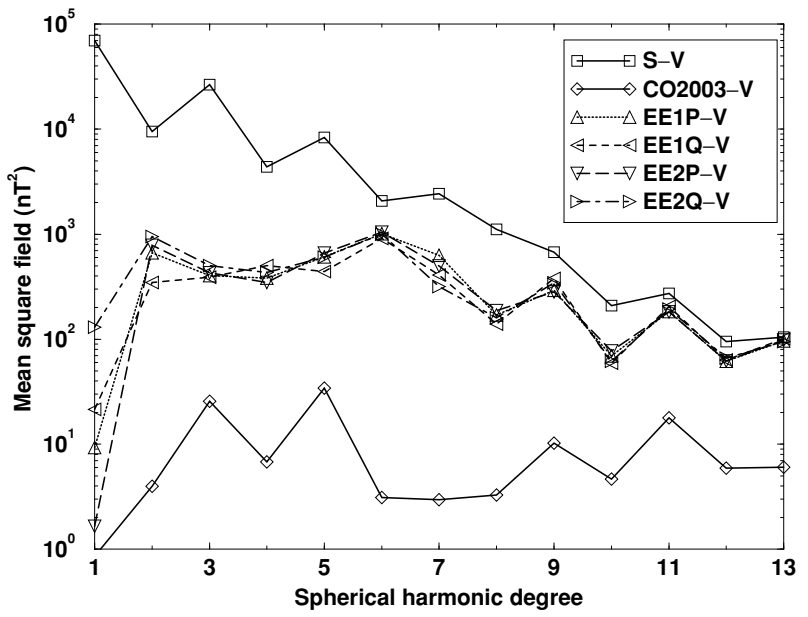

Fig. 4. Power spectra of difference of field models computed with electrojet peak locations from the vector data model, plotted at Earth radius $(r=6371.2 \mathrm{~km})$. The differences of model $\mathrm{V}$ from of model $\mathrm{S}$ and CO2003 are plotted from Fig. 2 for comparison. 

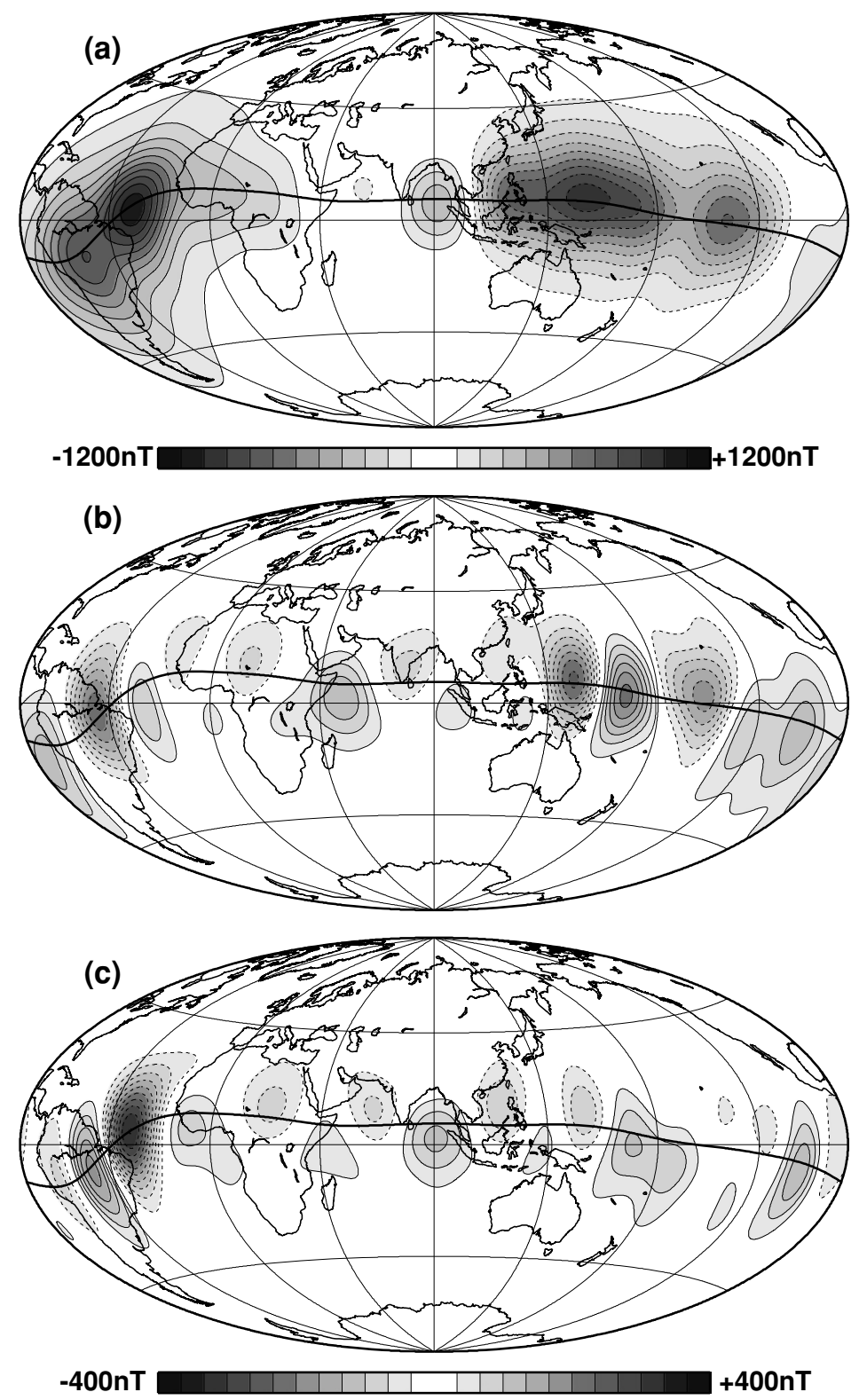

Fig. 5. Difference at $r=a$ between vertical field prediction of field model and that of model $\mathrm{V}$, for (a) model S (b) model EE1Q (c) model EECM. Dashed contours indicate negative values. Note the change in scale of the contour plot/gray shading: (a) has a contour interval of $100 \mathrm{nT}$, (b) and (c) have 1/3 the contour interval. The position of the magnetic dip equator at the Earth's surface (from CO2003) is also plotted.

another time of year, it is likely the models EE2P and EE2Q would have provided a better fit to model $V$ than EE1P and EE1Q. The data include signals from other ionospheric current systems, making up the SQ variation, which cannot be separated entirely from the electrojet, and which will disrupt the filtering process.

Several interesting points emerge from Table 1. While the inclusion of electrojet peak position information significantly improves model quality, the fit to the scalar data is almost unaffected. This is to be expected, as the electrojet peak positions can be fit by the addition or alteration of linear combinations of Gauss coefficients which are in the Backus series: in other words, they may be fit with little or no change in the model prediction of the scalar field. Also as a consequence of this, the models are insensitive to the choice of standard deviation for the two data sets (scalar and $Z=0$ at electrojet peak positions), as the two data sets are fit by almost independent linear combinations of Gauss coefficients. Note also that model V, although a much better representation of the field, fits the scalar data much less well than any of the other models. This is because vector data allow separation of internal and external field, and much of the misfit to the scalar data comes from from degree 1 external field, which we do not model. We do not estimate external field for model $\mathrm{S}$ or any of the electrojet models because this introduces further numerical instability related to the Backus effect (Holme and Olsen, in preparation), and a greater departure from model V/CO2003. The fit to the electrojet peak position data is similar for all EE models, and also similar in each case to the fit provided by model V.

Table 1 also includes diagnostics on two further models. EECM (Equatorial electrojet current model) is calculated with peak positions determined using the electrojet profile 
Table 1. Diagnostics of field models. Columns are RMS difference from model V (from CO2003 for model V), rms misfit to scalar data, and rms misfit to $0 \mathrm{nT}$ data at electrojet peak positions (in brackets, the same misfit for model V). Models EECM and EEPM are described later in the text.

\begin{tabular}{cccc}
\hline Model & RMS $(\mathrm{nT})$ & Misfit $(\mathrm{nT})$ & RMS at peak $(\mathrm{nT})$ \\
\hline S & 352.9 & 5.9 & \\
EE1P & 67.7 & 6.0 & $260(271)$ \\
EE1Q & 63.0 & 6.0 & $259(243)$ \\
EE2P & 68.2 & 6.0 & $259(259)$ \\
EE2Q & 69.7 & 6.0 & $262(235)$ \\
EECM & 56.6 & 6.1 & $216(239)$ \\
EEPM & 26.6 & 6.0 & \\
V & 11.21 & 10.24 & \\
\hline
\end{tabular}

of Lühr et al. (2004), determined at one degree longitude spacing (giving 360 data points). We might expect this model to provide optimal use of electrojet peak position information, but the improvement over our simple peakpicking methods is not great. Why this might be is shown in Fig. 5 in which we plot the difference at the Earth's surface from model $\mathrm{V}$ of model $\mathrm{S}$, model EE1Q (the best of the electrojet peak position models) and model EECM. The large departure of the field models near the dip equator is as expected, but as can be seen, the inclusion of dip equator information considerably improves the model. Note in particular that the contour interval in (b) and (c) is $1 / 3$ that for model $\mathrm{S}$ in (a). The large error in model EECM can be seen to be centred around $-45^{\circ}$ longitude. Examination of Fig. 3 shows that it is in this region that the fit of the Lühr et al. (2004) electrojet model to the dip equator is worst. The electrojet peak is much less clear (of lower amplitude and not as sharp) at this longitude. Whether the misfit of the model is physical (there is a departure of the electrojet from the dip equator) or observational (errors in the data or model) requires further study.

Finally, Table 1 also presents model EEPM (Equatorial electrojet perfect model) where the electrojet peak positions are synthesised at 720 positions separated by half a degree in longitude along the "true" dip equator from model CO2003. This model is representative of the best that could be reasonably expected from the method, if the electrojet provided perfect information on the magnetic dip equator. Comparing this model with model EE1Q thus gives a measure of to what extent inadequacies in the simple theory (that the local minimum in field magnitude at satellite altitude really does describe the position of the dip equator at $108 \mathrm{~km}$ height) and uncertainties in positioning degrade the modelling method.

The power spectra of the difference of EECM and EEPM from model $\mathrm{V}$ are plotted in Fig. 6 along with those of the reference model differences $\mathrm{S}-\mathrm{V}$ and $\mathrm{CO} 2003-\mathrm{V}$ and that of the best of the "peak position" models EE1Q. The improvement of the EECM model over the EE1Q model is entirely due to reduced power at degree 6. The EEPM model improves the model at all degrees, but it is difficult to compare the difference directly with that from model $\mathrm{V}$ due to the different total number of data in each case. Nevertheless, it is clear that better understanding of how the electrojet describes the dip equator would improve the field modelling - it is encouraging that at least the shape of the

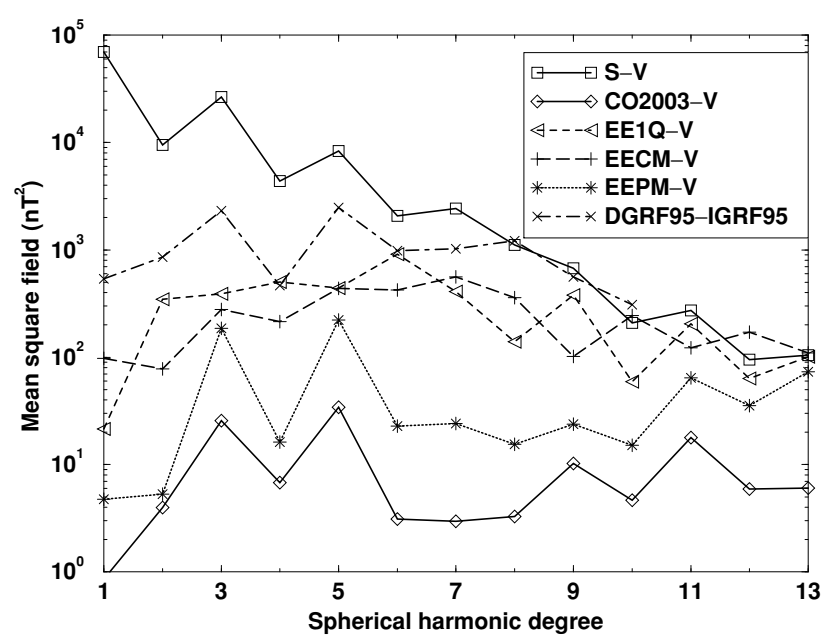

Fig. 6. Power spectra of difference of field models from vector data model $\mathrm{V}$, plotted at Earth radius $(r=6371.2 \mathrm{~km})$. Plotted are the scalar only model, the preferred dip equator peak position model, the model taking the electrojet profile of Lühr et al. (2004), and the vector data/CO2003 model departure. Also plotted is the difference between the IGRF1995 $7^{\text {th }}$ generation (Barton, 1997), and the recently adopted DGRF1995 (Macmillan et al., 2003) (these models are truncated at degree 10).

difference spectrum mimics closely that of model $\mathrm{V}$ from CO2003. Figure 6 includes one additional power spectrum. While we have shown that including information from electrojet peak positions strongly reduces the Backus effect, this does not provide any measure of whether the model is "useful" or not. To address this question, we examine the difference between the predictive 1995 IGRF $7^{\text {th }}$ generation (revised 1995) (Barton et al., 1996), and the DGRF 1995 model, recently adopted as definitive (Macmillan et al., 2003). To some extent the comparison is unfair: IGRF 1995 $7^{\text {th }}$ generation was a predictive model, and the data available for its modelling were known to be limited. Nevertheless, the field model obtained with satellite data only (and from only a six-day period) and using the constraint from electrojet peak positions, produces a better representation of the true field than the predictive IGRF model for 1995 did with all other available data.

\section{Discussion}

We have demonstrated that using observations of the position of the equatorial electrojet can greatly reduce the Backus effect. However, this information is clearly no re- 
placement for high-quality vector data. While satellites are flying which can return high-quality oriented vector data, such as Ørsted, CHAMP, or the proposed SWARM mission, this method for resolving Backus effect remains nothing more than a curiosity. However, if at some point in the future such a satellite is not available, the results argue very strongly for the occasional launch of a scalar-only satellite into a noon-midnight orbit, perhaps in the run up to the adoption of a new IGRF model. Such a satellite would require only a scalar magnetometer and GPS for positioning, and as a result, could be produced and run comparatively cheaply. The comparison with the IGRF model which was generated with limited satellite data (none at the epoch of the model) suggests that such a mission could be a very cost-effective method of producing an improved field model.

This study has been no more than a preliminary investigation. Should the need arise, clearly the modelling methodology could be improved by a better understanding of the electrojet, in particular whether it really departs from the dip equator over the Atlantic ocean, or whether this is a result of limited or poor data. Furthermore, the models presented here could be improved with additional data, particularly from observatories, particularly incorporated as secular variation, or with accurately determined crustal biases (Ultré-Guérard and Achache, 1997). Our method has one strong advantage in that the electrojet information covers all longitudes, and therefore even if of limited quality, such information could be a useful complement to other data sources.

However, whether or not the method is ever used in practice, we find it emotionally satisfying that, almost 40 years after the POGO mission, and despite the Backus effect, it is now in principal possible to construct an adequate model of the main magnetic field from satellite data consisting of intensity data alone.

Acknowledgments. We thank the CHAMP mission team, and especially Dr. M. Rother, for all their work in providing CHAMP data, without which this study would not have been possible. The contour plots were produced with GMT (Wessel and Smith, 1998).

\section{References}

Backus, G. E., Non-uniqueness of the external geomagnetic field determined by surface intensity measurements, J. Geophys. Res., 75, 63376341, 1970.

Barraclough, D. R. and C. E. Nevitt, The effect of observational errors on geomagnetic field models based solely on total-intensity measurements, Phys. Earth Planet. Int., 13, 123-131, 1976.

Barton, C. E., International Geomagnetic Reference Field: the seventh generation, J. Geomag. Geoelectr., 49, 123-248, 1997.

Barton, C. E., R. T. Baldwin, D. R. Barraclough, S. Bushati, M. Chiappini, Y. Cohen, R. Coleman, G. Hulot, P. Kotze, V. P. Golovkov, A. Jackson, R. A. Langel, F. J. Lowes, D. J. McKnight, S. Macmillan, L. R. Newitt, N. W. Peddie, J. M. Quinn, and T. J. Sabaka, International Geomagnetic Reference Field, 1995 edition, Geophys. J. Int., 125, 318-321, 1996.

Benkova, N. P., S. S. Dologinov, L. O. Tyurmina, and T. N. Cherevko, Comparison between the two satellite models, Izmiran and POGO, in World Magnetic Survey, 1957-1969, edited by A. J. Zmuda, vol. 28 of Bull. Int. Ass. Geomagn. Aeron., Paris, pp. 158-163, 1971.

Cain, J. C. and R. A. Langel, The geomagnetic survey by the polar orbiting geophysical observatories OGO-2 and OGO-4, 1965-1967, in World Magnetic Survey, 1957-1969, edited by A. J. Zmuda, vol. 28 of Bull. Int. Ass. Geomagn. Aeron., Paris, pp. 65-75, 1971.

Gubbins, D. and J. Bloxham, Geomagnetic field analysis-III. Magnetic fields on the core-mantle boundary, Geophys. J. R. Astron. Soc., 80,
695-713, 1985.

Holme, R. and N. Olsen, The spectrum of the magnetic secular variation, in Earth Observation with CHAMP-Results from Three Years in Orbit, edited by C. Reigber, H. Lühr, P. Schwintzer, and J. Wickert, pp. 329334, Springer Verlag, Berlin, 2004.

Hurwitz, L. and D. G. Knapp, Inherent vector discrepancies in geomagnetic field models based on scalar F, J. Geophys. Res., 79, 3009-3013, 1974.

Jadhav, G., M. Rajaram, and R. Rajaram, A detailed study of equatorial electrojet phenomenon using Ørsted satellite observations, J. Geophys. Res., 107, 10.1029/2001JA000183, 2002.

James, M. A., The Equatorial Electrojet: The Possible Implications of Locating it for Geomagnetic Field Modelling, Bachellors Thesis (unpublished), University of Liverpool, 2004.

Khokhlov, A., G. Hulot, and J.-L. Le Mouël, On the Backus effect-1, Geophys. J. Int., 130, 701-703, 1997.

Langel, R. A., Near-Earth magnetic disturbance in total field at high latitudes 1. Summary of data from OGO 2, 4 and 6, J. Geophys. Res., 79, 2363-2371, 1974.

Lowes, F. J., Spatial power spectrum of the main geomagnetic field, and extrapolation to the core, Geophys. J. R. Astron. Soc., 36, 717-730, 1974.

Lowes, F. J., Vector errors in spherical harmonic analysis of scalar data, Geophys. J. Roy. Astron. Soc., 42, 637-651, 1975.

Lowes, F. J. and J. E. Martin, Optimum use of satellite intensity and vector data in modelling the main geomagnetic field, Phys. Earth Planet. Int., 48, 183-192, 1987.

Lühr, H., S. Maus, and M. Rother, Noon-time equatorial electrojet: its spatial features as determined by the CHAMP satellite, J. Geophys. Res., 107, 10.1029/2002JA009656, 2004.

Macmillan, S., S. Maus, T. Bondar, A. Chambodut, V. Golovkov, R. Holme, B. Langlais, V. Lesur, F. Lowes, H. Lühr, W. Mai, M. Mandea, N. Olsen, M. Rother, T. Sabaka, A. Thomson, and I. Wardinski, The 9th-generation International Geomagnetic Reference Field, Geophys. J. Int., 155, 1051-1056, 2003.

Mandea, M. and S. Macmillan, International Geomagnetic Reference Field-the eighth generation, Earth Planets Space, 52, 1119-1124, 2000.

Mauersberger, P., Das Mittel der Energiedichte des geomagnetischen Hauptfeldes an der Erdoberfläche and seine säkulare Änderung, Gerlands Bietr. Geophys., 65, 207-215, 1956.

Maus, S., M. Rother, R. Holme, H. Lühr, N. Olsen, and V. Haak, First scalar magnetic anomaly map from CHAMP satellite data indicates weak lithospheric field, Geophys. Res. Lett., 29, 10.1029/2001GL013685, 2002.

Neubert, T., M. Mandea, G. Hulot, R. von Frese, F. Primdahl, J. L. Jorgensen, E. Friis-Christensen, P. Stauning, N. Olsen, and T. Risbo, Ørsted satellite captures high-precision geomagnetic field data, Eos Trans. $A G U, \mathbf{8 2}, 81-88,2001$.

Olsen, N., T. J. Sabaka, and L. Tøffner-Clausen, Determination of the IGRF 2000 model, Earth Planets Space, 52, 1175-1182, 2000.

Olsen, N., L. Tøffner-Clausen, T. J. Sabaka, P. Brauer, J. M. G. Merayo, J. L. Jørgensen, J.-M. Léger, O. V. Nielsen, F. Primdahl, and T. Risbo, Calibration of the Ørsted vector magnetometer, Earth Planets Space, 55, 11-18, 2003.

Onwumechili, C. A., The Equatorial Electrojet, Gordon and Breach, Amsterdam, 1997.

Reigber, C., H. Lühr, and P. Schwintzer, Champ mission status, Adv. Space Res., 30, 129-134, 2002.

Stern, D. P. and J. H. Bredekamp, Error enhancement in geomagnetic models derived from scalar data, J. Geophys. Res., 80, 1776-1782, 1975 .

Stern, D. P., R. A. Langel, and G. D. Mead, Backus effect observed by MAGSAT, Geophys. Res. Lett., 7, 941-944, 1980.

Ultré-Guérard, P. and J. Achache, Error analysis of total field models derived from POGS data, J. Geomag. Geoelectr., 49, 453-467, 1997.

Ultré-Guérard, P., M. Hamoudi, and G. Hulot, Reducing the Backus effect given some knowledge of the dip-equator, Geophys. Res. Lett., 25 , 3201-3204, 1998.

Voorhies, C. V., J. Santana, and T. Sabaka, Backus effect and perpendicular errors in harmonic models of real vs. synthetic data, IUGG 99 Abstracts, pp. B.388-B.389, 1999.

Wessel, P. and W. H. F. Smith, New, improved version of the generic mapping tools released, Eos Trans. AGU, 79, 579, 1998.

R. Holme (e-mail: holme@liv.ac.uk), M. A. James, and H. Lühr 